\title{
Une nouvelle arme pour vaincre la pharmacodépendance?
}

A près une rencontre initiale avec la drogue, la consommation de plus en plus répétée peut éventuellement conduire à un usage habituel, puis à un abus. La dépendance survient lorsque le malade drogué perd le contrôle de sa consommation, que son activité de recherche de drogue prend un caractère compulsif, et que des symptômes tels que la dysphorie, l'irritabilité ou encore une douleur physique apparaissent dans les périodes d'abstinence voulue ou forcée. Le malade drogué peut à tout moment interrompre cette évolution, mais ce qui caractérise la dépendance est souvent une succession de cycles de périodes d'abstinence ou de détoxification, suivies d'une rechute, c'est-à-dire d'un retour à l'usage compulsif de la drogue. Un des déterminants majeurs de la rechute est la mise en présence de stimulus liés à l'environnement dans le contexte de la prise de drogue. Par exemple, la simple vue d'une seringue ou la fréquentation des lieux habituels de consommation peuvent déclencher une envie irrésistible précipitant la reprise de la consommation. Les effets de ces éléments contextuels préalablement associés à la drogue, qui peuvent agir des années après l'interruption de la consommation, se manifestent aussi par des symptômes somatiques maintenant bien codifiés, tels qu'une tachycardie et une diminution de la température superficielle [1].

L'arsenal médicamenteux face à ce fléau est essentiellement fondé sur l'utilisation de produits de substitution, telles que la méthadone ou la buprénorphine $\left(\right.$ Subutex $\left.^{\circledR}\right)$ dans le cas de la dépendance aux opiacés ou des patches de nicotine pour le tabac. Ces produits constituent une aide à la détoxification, mais ne résolvent pas le problème de l'envie de drogue et tendent à perpétuer l'état de dépendance. Par ailleurs, ils sont d'un usage limité par leur spécificité liée au type de drogue consommée, et sont impuissants vis-à-vis des psychostimulants (cocaïne, amphétamines), qui, de plus en plus, prennent le pas sur des drogues comme l'héroïne. Une autre approche thérapeutique proposée s'efforce d'antagoniser l'effet de la drogue, en bloquant les récepteurs cérébraux impliqués (par exemple, la naltrexone pour les récepteurs opiacés [2]) ou en limitant son accès au cerveau (par exemple, avec des anticorps anti-cocaine [3]). Si cette approche peut être utile pour obtenir une détoxification rapide dans le cas d'une surdose, elle paraît d'une efficacité limitée dans un but prophylactique, car le malade drogué aura tendance à augmenter ses doses pour surmonter le blocage des effets de la drogue. L'idée même qu'une substance psychoactive puisse réduire l'envie et la recherche compulsive de drogue, sans être elle-même une drogue, n'avait pas encore été mise en pratique avant nos travaux récents [4].

L'hypothèse selon laquelle le récepteur $\mathrm{D}_{3}$ de la dopamine - que notre équipe avait découvert en 1990 [5] peut servir de cible pour des médicaments contre la toxicomanie est née d'une série d'observations convergentes. Le récepteur $\mathrm{D}_{3}$ est exprimé de manière plutôt sélective dans une sous-division cérébrale, l' "écorce » du noyau accumbens, qui est le lieu de projection des neurones dopami- nergiques issus de l'aire tegmentale ventrale. Or, la libération de dopamine par ces neurones est activée par toutes les drogues connues, bien que leur mode d'action primaire soit très différent. De plus, l'écorce du noyau accumbens est un "carrefour intégrant - en provenance de l'amygdale et de l'hippocampe - des informations sensorielles telles que celles engendrées par la présentation de stimulus préalablement associés à la prise de drogue [6]. L'expression du récepteur $\mathrm{D}_{3}$ a été trouvée anormalement élevée dans le noyau accumbens post-mortem de malades décédés de surdose de cocaïne [7], et il existe une association génétique entre le gène du récepteur $\mathrm{D}_{3}$ et la dépendance à l'héroïne [8]. Enfin, des agonistes et antagonistes dopamine1giques diminuent et augmentent respectivement l'auto-administration de cocaïne chez le rat entraîné, en corrélation avec leur sélectivité pour le récepteur $\mathrm{D}_{3}$ [9], mais il n'existait pas jusqu'alors d'agent hautement sélectif.

Ces éléments nous ont conduits, en collaboration avec l'équipe de Camille G. Wermuth (Cnrs ERS 1545, Strasbourg, France) à rechercher des molécules sélectives du récepteur $\mathrm{D}_{3}$. Nous avons sélectionné le BP 897, qui est un ligand très sélectif de ce récepteur, son affinité pour les autres sous-types de récepteurs de la dopamine étant au moins 100 fois inférieure. Sur des lignées cellulaires transfectées, le BP 897 se révèle comme un agoniste partiel du récepteur $\mathrm{D}_{3}$, capable de l'activer avec une efficacité égale à $60 \%$ de celle de la dopamine ou d'un de ses agonistes pleins (figure 1). Cette propriété nous est apparue intéressante dans le 


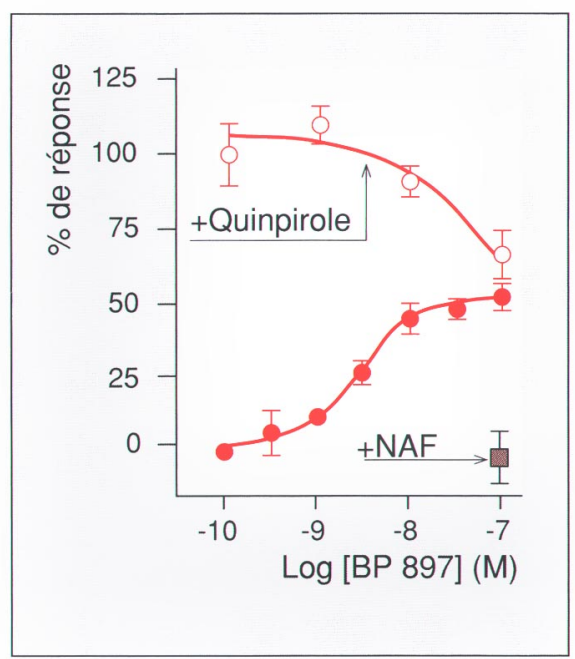

Figure 1. Agonisme partiel du BP 897. La stimulation par le BP 897 de cellules en culture exprimant le récepteur $D_{3}$ provoque une réponse (ici la mitogenèse), dont le maximum ne représente que $55 \%$ de la réponse maximale obtenue par un agoniste plein, le quinpirole. Cette réponse résulte bien du caractère agoniste du BP 897 parce qu'elle est antagonisée par le nafadotride (NAF), un antagoniste du récepteur $D_{3}$. Le BP 897 est aussi capable d'antagoniser partiellement l'effet du quinpirole. Grâce à cette dualité d'action, le BP 897 pourrait agir comme un régulateur de la transmission par le récepteur $D_{3}$ en compensant le manque la dopamine ou, au contraire, en réduisant les effets de l'excès de ce neuromédiateur.

contexte thérapeutique choisi, car les agonistes partiels avaient déjà été proposés pour lutter contre la dépendance [10]. La mesure de l'activité in vivo du $\mathrm{BP} 897$ sur le récepteur $\mathrm{D}_{3}$ a représenté une tâche plus difficile, car il n'existait pas de modèle animal formellement établi rendant compte de cette activité. Nous nous sommes assurés de la sélectivité du BP 897 visà-vis du récepteur $D_{3}$ dans des modèles animaux permettant le knock-in ou le knock-out de ce récepteur. Chez des rats dont les neurones dopaminergiques ont été détruits de manière unilatérale par une neurotoxine, l'expression du récepteur $\mathrm{D}_{3}$ est induite par des administrations répétées de lévodopa dans le stria- dans le contrôle de la motricité d'où ce récepteur est normalement absent. Le BP 897, en combinaison avec un agoniste du récepteur $D_{1}$, n'induit des rotations que chez les animaux chez lesquels l'expression $\mathrm{du}$ récepteur $\mathrm{D}_{3}$ a été induite par la lévodopa; cet effet du composé est donc dû à son activité d'agoniste du récepteur $\mathrm{D}_{3}$. Dans les îlots de Calleja, petites structures proches $\mathrm{du}$ noyau accumbens où le récepteur $\mathrm{D}_{3}$ est fortement exprimé, le BP 897, en combinaison avec un agoniste du récepteur $\mathrm{D}_{1}$, induit le gène $c$-fos chez des souris normales, mais pas chez des souris dont le gène du récepteur $\mathrm{D}_{3}$ a été génétiquement inactivé ; cet effet du BP 897 résulte cette fois d'une activité d'antagoniste. Ainsi, selon le neurone ou la réponse considérés, le BP 897 se comporte soit comme un agoniste, soit comme un antagoniste, ce qui est en accord avec ses propriétés d'agoniste partiel in vitro.

Un modèle chez le rat a été développé par l'équipe de Barry J. Everitt (Université de Cambridge, MA, USA), qui permet de mesurer l'effet d'une substance sur la recherche de cocaïne induite par un stimulus lumineux (figure 2). Le stimulus préalablement associé à la délivrance de la cocaïne a un effet démontrable sur le maintien du comportement de recherche de drogue et induit le rétablissement de ce comportement après son extinction [11], reflétant ainsi assez bien l'influence des stimulus liés à l'environnement sur la rechute chez les sujets dépendants. Le BP 897 s'est montré capable de réduire l'effet du stimulus lumineux sur la recherche de cocaïne, en l'absence de délivrance de la drogue, mais inefficace lorsque l'animal était placé sous l'emprise de la drogue

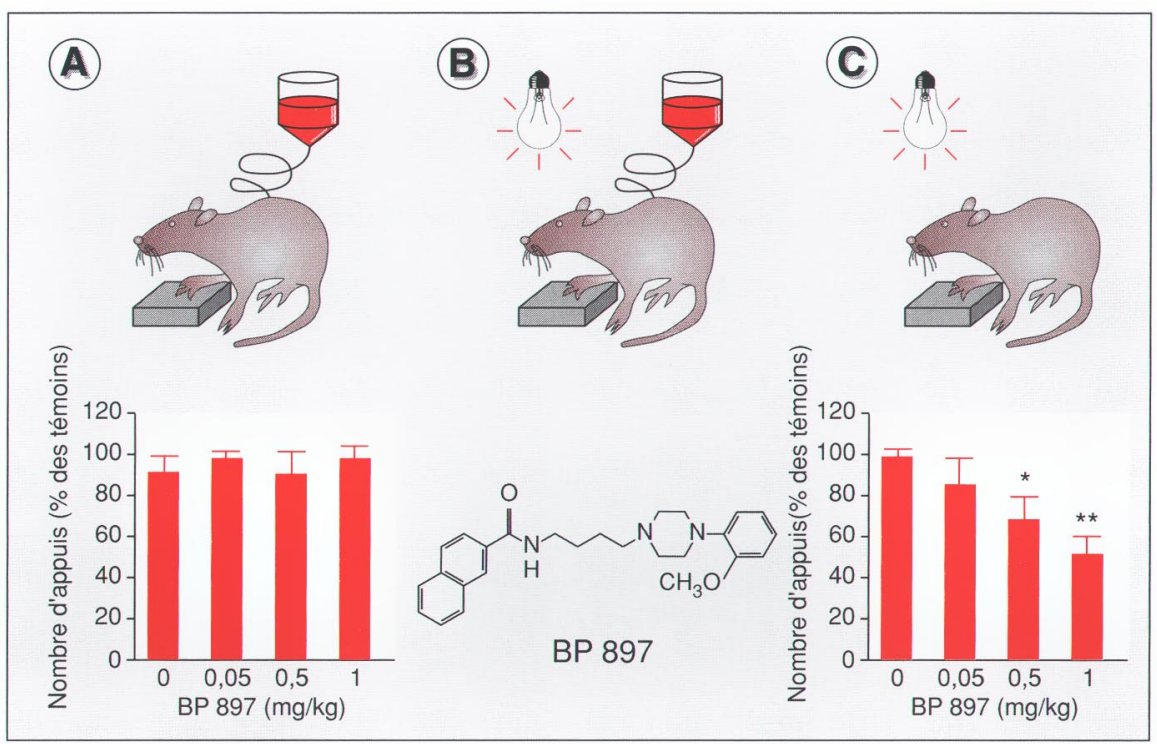

Figure 2. Un agoniste sélectif et partiel du récepteur $D_{3}$ (BP 897) réduit le comportement de recherche de cocaïne chez le rat, sans avoir de propriétés addictives. Lors de sessions régulièrement espacées, des rats sont entraînés à s'auto-administrer par voie intraveineuse de la cocaïne en appuyant sur un levier (A). Le BP 897, testé lorsque ce comportement s'est stabilisé au cours des sessions, ne modifie pas l'auto-administration de cocaïne, ce qui indique qu'il n'a pas de propriétés addictives. Par la suite, un stimulus neutre (un éclat lumineux) est associé à chaque auto-injection de drogue (B), et acquiert progressivement un pouvoir renforçant qui finalement maintient le comportement de recherche de cocaïne, même en l'absence de délivrance de la drogue (C). Durant cette dernière phase, le comportement de l'animal, qui reflète l'envie de drogue suscitée par le stimulus lumineux, est réduit de manière dépendante de la dose par le BP 897. ${ }^{*} p<0,05$ et ${ }^{*} p<0,01$ contre témoins par un test d'analyse de la variance (11 animaux par groupe). (D'après [4].) 
(figure 2). Il semble donc exister une dissociation entre l'effet du BP 897 sur la recherche de la drogue en période d'abstinence et un effet sur la prise de drogue, qui aurait alors reflété sa capacité de mimer les effets plaisants de la cocaïne ou de s'opposer à ses effets, comme le font les agonistes ou antagonistes classiques de la dopamine [9].

L'originalité du BP 897 par rapport aux agonistes ou antagonistes classiques de la dopamine s'est trouvée confirmée par le fait que cette substance n'est pas auto-administrée par des animaux entraînés à l'auto-administration de cocaïne. Il semble que cette propriété originale résulte à la fois de la spécificité du BP 897 vis-àvis du récepteur $D_{3}$ et de son caractère d'agoniste partiel, à mi-chemin entre agonistes et antagonistes induisant des effets opposés. En l'absence de stimulation du récepteur $\mathrm{D}_{3}$ par la dopamine, qui pourrait survenir dans les états d'abstinence, le BP 897 se comporte comme un agoniste en compensant le déficit en dopamine, mais sans entraîner une activation maximale qui alors aurait les mêmes conséquences que l'administration d'une drogue. Par ailleurs, la présentation de stimulus liés à l'environnement en rapport avec la drogue ont parfois les mêmes effets que l'administration de la drogue [1]. En s'opposant à l'activation du récepteur $\mathrm{D}_{3}$ par le stimulus, le BP 897 pourrait ainsi, par son activité d'antagoniste, exercer un effet «tampon», si bien que le sujet serait incapable de discerner le stimulus.

L'intérêt d'un tel mécanisme, encore hypothétique, est qu'il pourrait théoriquement opérer pour des stimulus associés à la prise de drogues ou de toxiques variés, comme l'héroïne ou la nicotine. L'utilisation de modèles animaux analogues à celui utilisé pour la cocaïne permettra de répondre à cette interrogation. Plus déterminants encore pour l'utilisation éventuelle du BP 897 dans la lutte contre la toxicomanie et le tabagisme seront les résultats d'une série d'essais cliniques qui débutera l'année prochaine

\section{RÉFÉRENCES}

1. Ehrman R, Robbins SJ, Childress AR, et $a l$. Conditioned responses to cocaine-related stimuli in cocaine abuse patients. Psychopharmacology 1992; 107 : 523-9.

2. Martin WR, Jasinski DR, Mansky PA. Naltrexone, an antagonist for the treatment of heroin dependence. Arch Gen Psychiatry 1973; 28 : 784-91.

3. Carrera MR, Ashley JA, Parsons LH, et al. Suppression of psychoactive effects of cocaine by active immunization. Nature $1995 ; 378: 666-7$.

4. Pilla M, Perachon S, Sautel F, et al. Selective inhibition of cocaine-seeking behaviour by a partial dopamine $\mathrm{D}_{3}$ receptor agonist. Nature $1999 ; 400: 371-5$.

5. Sokoloff P, Giros B, Martres MP, et al. Molecular cloning and characterization of a novel dopamine receptor $\left(\mathrm{D}_{3}\right)$ as a target for neuroleptics. Nature 1990; 347: 146-51.
6. Whitelaw RB, Markou A, Robbins TW, et al. Excitotoxic lesions of the basolateral amygdala impair the acquisition of cocaineseeking behaviour under a second-order schedule of reinforcement. Psychopharmacology $1996 ; 127$ : 213-24.

7. Staley JK, Mash DC. Adaptive increase in $\mathrm{D}_{3}$ dopamine receptors in the brain reward circuits of human cocaine fatalities. $\mathrm{J} \mathrm{Neu}$ rosci 1996 ; 16 : 6100-6.

8. Duaux E, Gorwood P, Sautel F, et al. Homozygosity at the dopamine $\mathrm{D}_{3}$ receptor gene is associated with opioid dependence. Mol Psychiatry 1998; 3 : 333-6.

9. Caine SB, Koob GF, Parsons LH, et al. $\mathrm{D}_{3}$ receptor functional test in vitro predicts potencies of dopamine agonists to reduce cocaine self-administration. NeuroReport $1997 ; 8: 2373-7$.

10. Pulverenti L, Koob GF. Dopamine agonists, partial agonists and psychostimulant addiction. Trends Pharmacol Sci 1994; 15: 374-9.

11. Arroyo M, Markou A, Robbins TW, et al. Acquisition, maintenance and reinstatement of intravenous cocaine self-administration under a second-order schedule of reinforcement in rats: effects of conditioned cues and continuous access to cocaine. Psychopharmacology 1999; 140 : 331-44.

\section{Pierre Sokoloff \\ Jean-Charles Schwartz}

Inserm U. 109, Unité de neurobiologie et de pharmacologie moléculaire, Centre Paul-Broca, 2 ter, rue d'Alésia, 75014 Paris, France.

\section{TIRÉS À PART}

P. Sokoloff. 